\title{
BMJ Open Observational study of the status of coronary risk biomarkers among Negritos with metabolic syndrome in the east coast of Malaysia
}

\author{
Atiqah Mokhsin, ${ }^{1}$ Siti Shuhada Mokhtar, ${ }^{1}$ Aletza Mohd Ismail, ${ }^{1}$ Fadzilah M Nor, ${ }^{1}$ \\ Syahrul Azlin Shaari, ${ }^{1}$ Hapizah Nawawi, ${ }^{2}$ Khalid Yusoff, ${ }^{3}$ Thuhairah Abdul Rahman, ${ }^{1}$ \\ Boon Peng Hoh ${ }^{3}$
}

To cite: Mokhsin A,

Mokhtar SS, Mohd Ismail A, et al. Observational study of the status of coronary risk biomarkers among Negritos with metabolic syndrome in the east coast of Malaysia. BMJ Open 2018;8:e21580. doi:10.1136/ bmjopen-2018-021580

- Prepublication history for this paper is available online. To view these files, please visit the journal online (http://dx.doi org/10.1136/bmjopen-2017021580).

Received 11 January 2018 Revised 2 October 2018 Accepted 5 October 2018

Check for updates

(c) Author(s) (or their employer(s)) 2018. Re-use permitted under CC BY-NC. No commercial re-use. See rights and permissions. Published by BMJ.

${ }^{1}$ Faculty of Medicine, Universiti Teknologi MARA, Sungai Buloh, Selangor, Malaysia

${ }^{2}$ Institute of Pathology, Laboratory and Forensic

Medicine (I-PPerForM), Universiti Teknologi MARA, Sungai Buloh, Selangor, Malaysia

${ }^{3}$ Faculty of Medicine and Health Sciences, UCSI University,

Cheras, Kuala Lumpur, Malaysia

Correspondence to Dr Thuhairah Abdul Rahman; thuhairah@salam.uitm.edu.my and Dr Boon Peng Hoh;

hohbp@ucsiuniversity.edu.my

\section{ABSTRACT}

Objectives To determine the prevalence of metabolic syndrome (MS), ascertain the status of coronary risk biomarkers and establish the independent predictors of these biomarkers among the Negritos.

Settings Health screening programme conducted in three inland settlements in the east coast of Malaysia and Peninsular Malaysia.

Subjects 150 Negritos who were still living in three inland settlements in the east coast of Malaysia and 1227 Malays in Peninsular Malaysia. These subjects were then categorised into MS and non-MS groups based on the International Diabetes Federation (IDF) consensus worldwide definition of MS and were recruited between 2010 and 2015. The subjects were randomly selected and on a voluntary basis.

Primary and secondary outcome measures This study was a cross-sectional study. Serum samples were collected for analysis of inflammatory (hSCRP), endothelial activation (sICAM-1) and prothrombogenesis [Ip(a)] biomarkers.

Results MS was significantly higher among the Malays compared with Negritos (27.7\%vs12.0\%). Among the Malays, MS subjects had higher hsCRP $(p=0.01)$ and sICAM-1 $(p<0.05)$ than their non-MS counterpart. There were no significant differences in all the biomarkers between MS and the non-MS Negritos. However, when compared between ethnicity, all biomarkers were higher in Negritos compared with Malays $(p<0.001)$. Binary logistic regression analysis affirmed that Negritos were an independent predictor for $L p(a)$ concentration $(p<0.001)$. Conclusions This study suggests that there may possibly be a genetic influence other than lifestyle, which could explain the lack of difference in biomarkers concentration between MS and non-MS Negritos and for Negritos predicting $\mathrm{Lp}(\mathrm{a})$.

\section{INTRODUCTION}

Orang Asli (OA) are the indigenous people believed to be the earliest inhabitants of Peninsular Malaysia. There are 18 OA tribes, categorised under three main groups according to their different languages and
Strengths and limitations of this study

- This study gains access to the earliest and smallest population of the Malaysian aboriginal tribe, the inland dwelling Negrito, whom have completely isolated themselves from influences of modernisation.

- This is one of the first studies that assessed biomarkers of atherogenesis among the inland-dwelling Negritos other than hsCRP.

- This is one of the first studies to determine the independent predictors of the atherogenesis biomarkers among inland-dwelling Negritos.

- The small sample size of Negritos due to difficulties accessing remote tribes and small total population poses a limitation despite achieving a minimum sample size requirement.

customs: Negritos, located in the northern part of the peninsula; Senoi, residing in the Central Region and Proto-Malays (or Aboriginal Malay) living in the southern region. The OA constitutes approximately $0.5 \%$ (150 000 ) of the total Malaysian population with Senoi representing the largest OA population (54\%) followed by Proto Malays $(43 \%)$ and Negritos (3\%). ${ }^{1}$

Negrito, known to be the earliest OA tribe to arrive in Peninsular Malaysia, is believed to have occupied this region of Malaysia approximately 25000 years ago. ${ }^{2}$ A substantial group of the Negritos continue to practice nomadic lifestyle for reasons such as illness, food resources and intra-tribal feuds.

The poverty rate among $\mathrm{OA}$ is $76.9 \%$ with the majority of OA living in the jungles or rural areas, while a minority have moved into urban areas. ${ }^{3}$ Although the Malaysian government has taken measures to eradicate the poverty level among the OAs, which subsequently led to the reduction of poverty-associated diseases such as malaria, tuberculosis, 
AIDS and dental decay, ${ }^{3}$ to name a few, disorders such as metabolic syndrome (MS) and coronary artery disease $(\mathrm{CAD})$ are not well addressed. This could most likely stem from the common misconception that these are 'diseases of the rich', when in fact, it is not exclusive to one type of socioeconomic group but transcends all walks of life. ${ }^{45}$

MS, a major public health challenge, is a cluster of metabolic disturbances that result from a complex interaction between genetic and environmental factors. ${ }^{6}$ MS is associated with increased risk of $\mathrm{CAD}^{7}{ }^{7}$ The metabolic abnormalities that underlie the definition of MS include insulin resistance, central obesity, dyslipidaemia, hypertension and glucose intolerance. ${ }^{8}$ The prevalence of MS among Malaysians was estimated to be between $37.1 \%$ and $42.5 \%$ in $2008 .^{9}$

It has also been well established that MS is associated with enhanced inflammation, endothelial activation and prothrombogenesis that are the key processes in atherosclerosis. Previous studies have documented the association between MS and enhanced inflammation. ${ }^{10}$ Increases in proinflammatory cytokines such as interleukin-6, resistin and C-reactive protein are due to the overproduction by monocyte-derived macrophages residing in the expanded adipose tissue mass. ${ }^{11}$ The National Cholesterol Education Program - Adult Treatment Panel (NCEP-ATP) reported higher soluble endothelial adhesion molecules such as intercellular adhesion molecule-1, vascular adhesion molecule-1 and E-selectin among subjects with MS. ${ }^{12}$ Furthermore, various studies have also shown elevated lipoprotein (a) $[\mathrm{Lp}(\mathrm{a})]$ in MS subjects. ${ }^{13} \mathrm{Lp}(\mathrm{a})$ has been found to significantly reduce endogenous clot lysis in plasma ex vivo, ${ }^{14}$ possibly to its binding to fibrinogen and attenuating fibrin-mediated enhancement of tissue plasminogen activator of plasminogen. ${ }^{15}$

Despite MS becoming a major public health concern with extensive data on this syndrome worldwide, reports on its prevalence in Malaysia remain scarce, particularly so among the OA in the country. There have been previous studies addressing factors related to MS globally in Malaysia ${ }^{16}$; however, the samples used in these studies were not a representative of the Malaysian population as they focused on the major ethnicities in Malaysia - Malay, Chinese and Indian, while the OA population was poorly represented.

To the best of our knowledge, there is only one recent published study that reported the urbanised Orang Seletar (a Proto-Malay subtribe) having the highest prevalence for central obesity $(66.1 \% \pm 5.9 \%)$ compared with the other subtribes who are less urbanised (Senoi and Negrito). The study also revealed that the prevalence of hypertension was highest among the Negritos $(43.8 \% \pm 9.3 \%$ and $51.2 \% \pm 15.3 \%)$ who resided in most remote areas and were the leanest among the six OA subtribes investigated. ${ }^{1}$

The lack of data on the prevalence of MS among OA, specifically the Negritos, could most likely stem from the preconceived notion that they are not susceptible to the disorder due to their detachment from urbanisation and their healthier lifestyle. Furthermore, there have been very few studies investigating the status of the biomarkers reflecting inflammation, endothelial activation and prothrombogenesis among the Negritos with MS. In addition, determining the MS components among them will further identify potential modifiable coronary risks such as hypertension, smoking, dyslipidaemia and glucose intolerance through proper education and healthcare services.

Therefore, this study aims to determine the MS components among Negrito, identify their status of inflammation, endothelial activation and prothrombogenesis and ascertain the independent predictors for these biomarkers of coronary risk.

\section{SUBJECTS AND METHODS}

\section{Target population and sample collection}

One hundred and fifty Negrito subjects were recruited in this cross-sectional study. They were from Bateq and Mendriq sub-tribes, from three inland settlements in Gua Musang, Kelantan, the east coast of Peninsular Malaysia $\left(4.8843^{\circ} \mathrm{N}, 101.9682^{\circ} \mathrm{E}\right)$. Also, 1177 Malays were recruited from various national health screening programmes in Peninsular Malaysia. The subjects were randomly selected and on a voluntary basis. These subjects were then categorised into MS and Non-MS groups based on the IDF's consensus worldwide definition of MS. ${ }^{17}$ All methods were carried out in accordance with relevant guidelines and regulations.

The sample size was calculated using PS Power and Sample Size Calculations V.3. $0^{18}$ with a power of study at 99\% and prevalence of MS among Negritos and Malays at $15.2 \% \pm 5 \%{ }^{19}$ and $38.8 \% \pm 5 \%{ }^{20}$ respectively. The minimum sample size calculated for Negritos and Malays are both 125. Due to the significant sample size difference between the two ethnic groups recruited and the relative difficulty in accessing the Negrito's remote locations of habitat, it is relevant to highlight that this study conducted a within-ethnic group rather than a between-group comparison of the biomarkers of interest when subdivided between MS and non-MS.

Demographic data was gathered by interview questionnaire. Information such as age, gender, tribe, education and occupation, health-related questions such as subjects' past medical history and social history including smoking status were recorded. Family history of cardiometabolic and infectious diseases were also recorded.

Topography measurements included blood pressure (BP), body mass index (BMI)), waist circumference (WC) and waist-to-hip ratio. With the subject in a seated position and following 5-10 min of rest, BP was measured by an automated BP reader (cuff size $12 \times 33 \mathrm{~cm}$, Colin press-mate, Japan). The systolic BP and diastolic BP were measured to the nearest $1 \mathrm{~mm} \mathrm{Hg}$. BMI was calculated using the formula: $\mathrm{BMI}=$ weight $(\mathrm{kg}) / \mathrm{height}^{2}\left(\mathrm{~m}^{2}\right)$. WC was measured to the nearest $0.5 \mathrm{~cm}$ using a measuring tape at midway between the inferior margin of the last rib and 
the iliac crest in a horizontal plane. Hip circumference measurement was taken around the pelvis at the point of maximal protrusion of the buttocks. Any visible stigmata of dyslipidaemia and diabetes mellitus were documented.

\section{Defining MS (IDF Criteria, 2006)}

An individual was classified as having MS if central obesity was exhibited along with at least two of the following: (1) elevated triglyceride (TG) concentration of $>1.7 \mathrm{mmol} / \mathrm{L}$, (2) reduced high-density lipoprotein cholesterol (HDL-c) of $<1.0 \mathrm{or} 1.3 \mathrm{mmol} / \mathrm{L}$ in male and female respectively, (3) elevated $\mathrm{BP}$ of $>140 / 90 \mathrm{~mm} \mathrm{Hg}$, and a raised fasting plasma glucose of $\geq 5.6 \mathrm{mmol} / \mathrm{L}$. Central obesity, using the suggested WC for Asian/South Asians, was defined as $\geq 90 \mathrm{~cm}$ and $\geq 80 \mathrm{~cm}$ for males and females respectively. ${ }^{17}$

\section{Venous blood sampling and on-site biochemical analysis}

Venous blood samples were collected following a non-traumatic venepuncture between 0800 hours and 1500 hours. Serum and plasma were separated by centrifugation at $3500 \mathrm{rpm}$ for $10 \mathrm{~min}$ within 1 hour and stored at $-20^{\circ} \mathrm{C}$ before analyse.

\section{Biochemical analysis}

Serum samples were sent to Centre for Pathology Diagnostic \& Research Laboratories of Faculty of Medicine, Universiti Teknologi MARA, Selangor, Malaysia. All clinical chemistry tests analysed were MS ISO 15189:2014 accredited.

Cardiometabolic parameters tested were fasting serum lipids including total cholesterol, TG and HDL-c, which were measured by enzymatic reference methods. Plasma glucose was analysed by hexokinase method. All methods were run on an automated analyser (Cobas Integra 400 PLUS, Roche Diagnostic, Germany) except for low-density lipoprotein cholesterol concentration, which was derived using the Friedewald equation. ${ }^{21}$

\section{Analyses of biomarkers of inflammation, endothelial activation and prothrombogenesis}

The biomarkers analysed included hsCRP, sICAM- 1 and $\mathrm{Lp}(\mathrm{a})$. Both hsCRP and $\mathrm{Lp}(\mathrm{a})$ were measured using turbidimetric method on an automated analyser (Cobas Integra 400 PLUS, Roche Diagnostics, Germany). Serum s-ICAM-1 concentration was determined by enzymelinked immunosorbent assay based on the measured optical density (eBioscience Bender MedSystems, Vienna Austria).

\section{Data analysis}

The associations of all three biomarkers were compared between two groups of MS and non-MS, using independent t-test, SPSS V.20. Binary logistic regression was performed on the independent variables (coronary risk biomarkers) to assess their impact on the likelihood that subjects would fall into each of the MS component (obesity, hypertension, diabetes mellitus, and low HDL-c or high TG concentrations). The specific model estimated from the data was: logit (coronary risk biomarkers) $=\alpha+\mathrm{b} 1($ age $)+\mathrm{b} 2($ ethnicity $)+\mathrm{b} 3$ (gender) $+\mathrm{b} 4$ (smoking status) +b5 (BMI) +b6(WC) +b7 (SBP) +b8 (DBP) +b9 (glucose concentration) +b10 (HDL-c concentration) +b11 (TG concentration), where the dependent variable is logit coronary risk biomarkers, $\alpha$ is the estimate for the intercept and b1, b2,..,b11 are estimates for the coefficients of the 11 predictors.

The variables were represented by two dummy variables to reflect the number of responses and reference categories. They were: ethnicity=Malay (1), Negrito (0), Negrito=reference group; gender, female (1), male $(0)=$ reference group; smoking status, smoking (1) and not smoking $(0)=$ reference group; While for age, BMI, WC, SBP, DBP, glucose, HDL-c and TG concentrations were continuous variables.

\section{Patient and public involvement}

Patients were not involved in the recruitment or conduct of the study. Written, informed consent was obtained from all subjects aged 18 years and above, prior to recruitment. Communications were done in Bahasa Malaysia with some input from local translators, either the Department of Orang Asli Development (JAKOA) officers or tribal leaders if and when required. The results of this study were disseminated to subjects through the JAKOA officers or using the postal address if provided. A physician was placed in the settings during the health screening programmes to provide an advice or referral letter when necessary.

\section{RESULTS}

\section{Demographic data}

Table 1 summarises the demographic parameters for the Malays and Negritos. A total of 326 Malays $(27.7 \%)$ and 18 Negritos (12.0\%) were diagnosed with MS based on the IDF 2006 criteria. On a separate note, among the Malays, $15.5 \%$ of them had diabetes while $1.5 \%$ of the Negritos had IFG $\geq 5.6 \mathrm{mmol} / \mathrm{L}$ or random plasma glucose of $>11.0 \mathrm{mmol} / \mathrm{L}$. We observed that $43.9 \%$ of the Malays and $57.2 \%$ of the Negritos suffered from hypertension while $58.7 \%$ Malays and $14.0 \%$ Negritos had central obesity.

Compared with the non-MS Negritos, their MS counterpart had higher TC $(5.30 \pm 1.05$ vs $4.48 \pm 0.85 \mathrm{mmol} / \mathrm{L}, \mathrm{P}<0.001$ respectively), TG $(2.14 \pm 0.79$ vs $1.41 \pm 0.68 \mathrm{mmol} / \mathrm{L}, \mathrm{P}<0.001$ respectively) and LDL-c (3.36 \pm 0.69 vs $2.80 \pm 0.84 \mathrm{mmol} / \mathrm{L}, \mathrm{P}=0.008$ respectively). The most frequent criteria diagnosing MS among the Negritos were hypertension, elevated TG and low HDL-c and none of the Negritos diagnosed with MS had any form of glucose intolerance.

\section{Comparison of the biomarkers of inflammation, endothelial activation and prothrombogenesis in Malay and Negrito subjects}

As can be seen from table 2 there was no significant differences between MS and non-MS among 
Table 1 Demographic and clinical characteristics of the Negritos and Malays

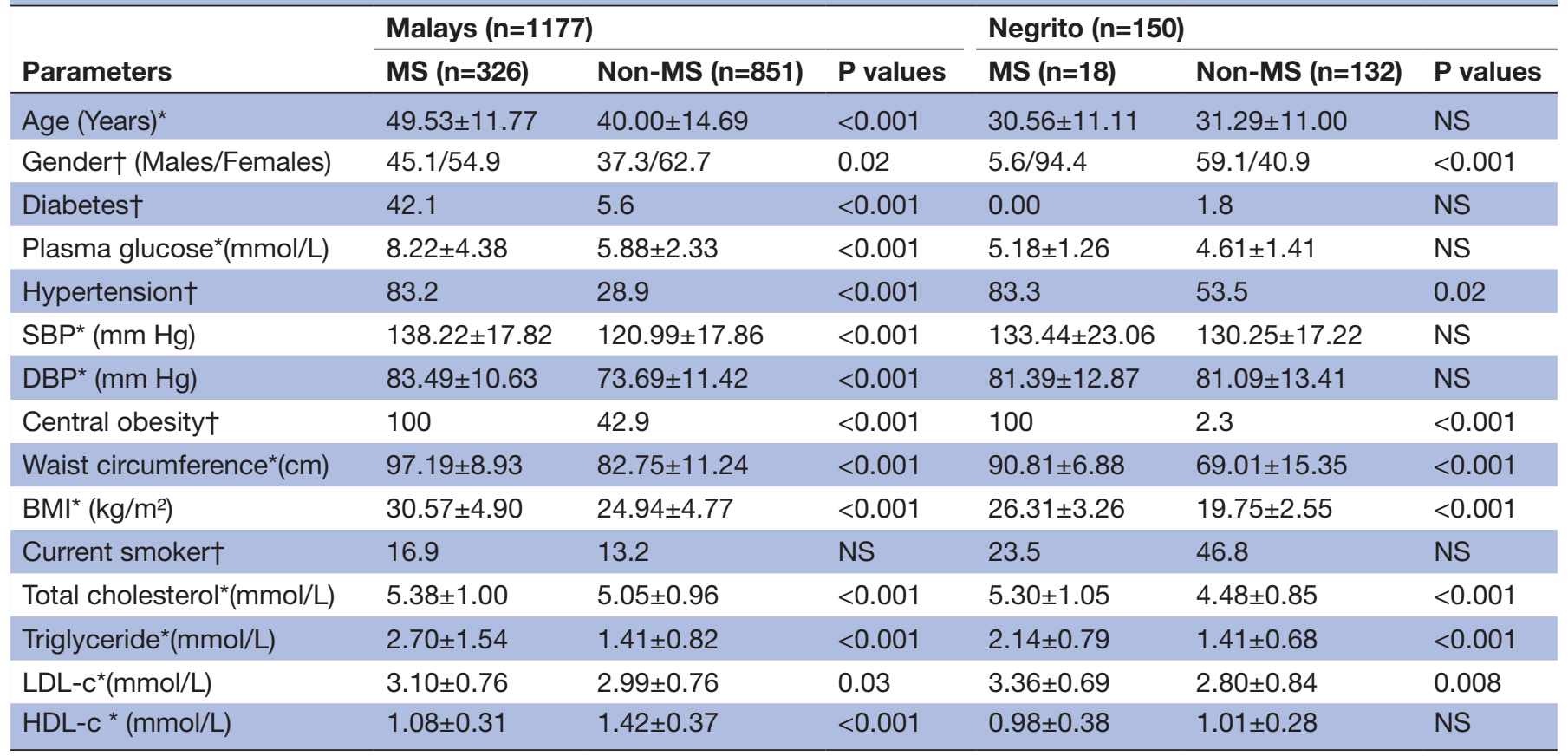

${ }^{*}$ Data expressed as mean $\pm S D$. $p$-value was significant at $p<0.05$, T-test was applied.

† Data expressed as percentage. $p$-value was significant at $p<0.05$, chi-square test was applied.

BMI, body mass index; DBP, diastolic blood pressure; NS, not significant; SBP, systolic blood pressure.

Negritos for all biomarkers $(\mathrm{P}>0.05)$ whereas MS Malays illustrated higher hsCRP (mean $\pm \mathrm{SD}=1.88 \pm 1.16$ vs $1.26 \pm 1.13 \mathrm{mg} / \mathrm{L}, \mathrm{p}<0.001$ respectively) and sICAM-1 (mean $\pm \mathrm{SD}=531.73 \pm 185.73$ vs $465.46 \pm 202.08 \mathrm{ng} / \mathrm{mL}$, $\mathrm{p}<0.001$ respectively) concentrations compared with their non-MS counterpart. When comparing between Negritos and Malays, Negritos showed significantly higher concentrations of hsCRP (mean $\pm \mathrm{SD}=2.27 \pm 2.25$ vs $\quad 1.41 \pm 1.17 \mathrm{mg} / \mathrm{L}, \quad \mathrm{p}<0.001$ respectively), $\quad$ sICAM-1 (mean $\pm \mathrm{SD}=684.85 \pm 388.03$ vs $482.25 \pm 200.05 \mathrm{ng} / \mathrm{mL}$, $\mathrm{p}<0.001$ respectively) and $\mathrm{Lp}(\mathrm{a})$ (mean $\pm \mathrm{SD}=0.22 \pm 0.22$ vs $0.06 \pm 0.04 \mathrm{mg} / \mathrm{L}, \mathrm{p}<0.001$ respectively) compared with the Malays.

Independent predictors of the biomarkers of inflammation, endothelial activation and prothrombogenesis in Malay and Negrito subjects

To further determine the independent predictors of these biomarkers, binary logistic regression analyses were performed with the biomarkers as dependent variables (table 3). It was found that BMI $(p=0.016)$, FPG $(p<0.001)$ and DBP $(p=0.042)$ were independent predictors for hsCRP while the Negrito determined $\operatorname{Lp}(\mathrm{a}) \quad(\mathrm{p}<0.001)$ when taken account all subjects after adjusting for age, gender, DM, hypertension, smoking status and BMI.

Among the Malays, it was found that male $(p<0.001)$, BMI $(p<0.001)$ and FPG concentration $(p<0.001)$ predicted for hsCRP while male $(p=0.040)$ and FPG $(\mathrm{p}=0.039)$ predicted $\operatorname{Lp}(\mathrm{a})$ after adjusting for the same parameters. There were no independent predictors for any of these biomarkers among Negritos.

\section{DISCUSSION}

MS is defined by an aggregation of atherosclerotic factors, mainly central obesity, hypertension, glucose intolerance and dyslipidaemia, which, in combination, enhance the probability of developing type $2 \mathrm{DM}$ and $\mathrm{CAD}{ }^{22}$ In two European studies, ${ }^{23} 24$ the presence of MS predicted

Table 2 Concentrations of inflammatory, endothelial activation and prothrombogenesis biomarkers in MS and non-MS Malays and Negritos

\begin{tabular}{|c|c|c|c|c|c|c|}
\hline \multirow[b]{2}{*}{ Biomarkers } & \multicolumn{3}{|l|}{ Malays $(n=1177)$} & \multicolumn{3}{|l|}{ Negrito $(n=150)$} \\
\hline & MS (n=326) & Non-MS $(n=851)$ & P values & MS (n=18) & Non-MS $(n=132)$ & $P$ values \\
\hline hsCRP (mg/L) & $1.88 \pm 1.16$ & $1.26 \pm 1.13$ & $<0.001$ & $4.21 \pm 3.20$ & $2.06 \pm 2.04$ & NS \\
\hline Lp(a) (g/L) & $0.06 \pm 0.04$ & $0.06 \pm 0.04$ & NS & $0.23 \pm 0.26$ & $0.22 \pm 0.22$ & NS \\
\hline
\end{tabular}

Data expressed as mean $\pm S D$. $p$-value was significant at $p<0.05$, T-test was applied.

NS, not significant. 


\begin{tabular}{|c|c|c|c|c|c|}
\hline Variables & Independent predictor & Beta & Adjusted OR & 95\% CI Lower, Upper & $P$ values \\
\hline hsCRP & 1. $\mathrm{BMI}\left(\mathrm{kg} / \mathrm{m}^{2}\right)$ & 0.05 & 1.05 & 1.01 to 1.10 & 0.016 \\
\hline & 3. Diastolic (mm Hg) & 0.01 & 1.01 & 1.00 to 1.02 & 0.042 \\
\hline $\operatorname{Lp}(a)$ & 1. Ethnicity (Negrito) & 1.03 & 2.79 & 1.87 to 4.14 & $<0.001$ \\
\hline & 2. $\mathrm{BMI}\left(\mathrm{kg} / \mathrm{m}^{2}\right)$ & 0.18 & 1.20 & 1.16 to 1.24 & $<0.001$ \\
\hline & 3. Glucose (mmol/L) & 0.08 & 1.08 & 1.04 to 1.13 & $<0.001$ \\
\hline $\operatorname{Lp}(\mathrm{a})$ & 1. Gender (Male) & -0.56 & 0.57 & 0.39 to 0.84 & 0.004 \\
\hline & 2. Glucose (mmol/L) & -0.08 & 0.93 & 0.86 to 1.00 & 0.039 \\
\hline
\end{tabular}

The model reasonably fits well. Model assumptions are met. There are no interaction and multicollinearity problem.

increased CAD mortality. These findings are not unexpected considering MS comprises established risk factors for CAD such as hypertension, impaired glucose tolerance, low HDL-c concentration, elevated TG concentration and obesity, which enhances endothelial activation and inflammation, key processes in atherogenesis. Furthermore, coronary risk biomarkers are strongly associated with endothelial dysfunction ${ }^{25}$ and thrombosis ${ }^{26}$ and are elevated in patients with atherosclerosis-related disease such as $\mathrm{CAD}^{27}$ and peripheral artery disease ${ }^{28}$ in previous reports.

There have been several previous reports determining the prevalence of non-communicable diseases (NCDs) such as DM, hypertension, dyslipidaemia and obesity among OA subjects. A previous report on the health status of the Negrito subtribe Lanoh showed that $8.9 \%$ were recently diagnosed with hypertension, $6.7 \%$ had hypertension and DM and an alarming $26.7 \%$ had pre-obesity. ${ }^{29}$ A larger population study done identified central obesity and hypertension among OA sampled from seven different subtribes and eight settlements. ${ }^{1}$ What is more alarming is the report by Aziz et al, which highlighted the higher percentage of OA having high insulin levels, hsCRP and higher percentage categorised as high risk by the Framingham Risk Score, which forecasts 10-year risk of CAD. ${ }^{30}$ These reports strongly imply that NCDs such as hypertension, dyslipidaemia, central obesity and DM, which are clusters of metabolic factors associated with MS, have gradually led to the increase in CAD risk among OA.

Although several reports highlighted the increasing prevalence of NCDs among the OA in Malaysia, there is scarce research that extended to investigate biomarkers for coronary risk among this population. To the best of our knowledge, there is only one other study which reported the status of inflammatory biomarker among OA in Peninsular Malaysia compared with Malays. ${ }^{30}$ Our study further explored other biomarkers reflecting atherogenesis such as endothelial activation and prothrombogenesis. This study showed that biomarkers of inflammation (hsCRP) and endothelial activation (sICAM-1) were enhanced in MS Malays compared with their non-MS counterpart.
These findings are expected and in keeping with previous studies denoting the higher CAD risk among MS. ${ }^{17} 30$ The association of MS with inflammation and endothelial activation is also well documented. ${ }^{12} 31$ The elevated sICAM-1 seen among MS Malays could be attributed to higher prevalence of hypertension, obesity and dyslipidaemia compared with their non-MS counterpart, which is parallel with previous reports. ${ }^{31} 32$

However, we were unable to observe any statistical difference in the concentrations of biomarkers of inflammation, endothelial activation and prothrombogenesis between MS and non-MS among the Negritos. This contradicts previous reports underscoring enhanced inflammation among OAs. ${ }^{30}$ These inconsistent findings could be attributed to the small sample size of Negritos in comparing the biomarkers, although the minimum sample size calculated was achieved. Furthermore, a previous study conducted to determine the prevalence of MS in an OA population found that the prevalence of MS among inland Negritos to be $12.5 \%$, which is consistent with our findings of $12 \%$ among our Negrito population. ${ }^{19}$ The population differences between the previous study and ours could also have led to these differences, where we recruited mainly inland Negritos in the northern state of Malaysia where majority of them are distributed, while the previous study recruited OAs from a mixture of inland and peripheries of towns where urbanisation could have influenced lifestyle, which could enhance inflammation and insulin resistance. ${ }^{30}$

In addition, as a general index of inflammation, CRP concentrations have been shown to vary by ethnicity and within ethnic groups by fitness, as it was reported to be higher in healthy Indian Asians than in European white people and were related to greater central obesity and insulin resistance in Indian Asians. ${ }^{33}$ Furthermore, none of the Negritos diagnosed with MS had any form of glucose intolerance, which could possibly explain these inconsistent findings as there have been reports on the association between CRP and sICAM-1 with MS, hypertension, and DM. ${ }^{34}{ }^{35} \mathrm{It}$ is also worth highlighting that when comparing these 
biomarkers between Negritos and Malays, we observed higher concentrations of all three biomarkers among the younger aged Negrito subjects compared with the Malays. This suggests that the Negritos may be at higher risk of CAD at a younger age group, hence warranting further investigation.

Furthermore, findings from this study suggest that the MS Negritos despite having coronary risk factors such as hypertension and dyslipidaemia, the failure to exhibit enhanced atherogenesis compared with their non-MS counterpart could possibly be attributed to genetic and/or lifestyle influences, which could play a role in attenuating atherogenesis. The Negritos included in this study were located in remote areas of northern Peninsular Malaysia and to certain extent, still practising the hunter-gatherer lifestyle and living isolated from urbanisation thus may have contributed to the differences observed in the biomarkers between the two ethnicities when comparing with the MS and non-MS counterparts. This is in agreement with previous studies, which reported improved inflammatory endothelial activation and prothrombogenesis status in MS subjects, following aggressive lifestyle modification, which included dietary improvement and initiation and maintenance of exercise. ${ }^{36} 37$

One possible postulating genetic factor that may contribute to this finding is the genetic variation of the candidate gene $\mathrm{CDH} 13$. Recent population genomic studies on the Negritos have identified a strong and consistent positive natural selection signal spanning the genomic region which harbours $C D H 13 .{ }^{38}{ }^{39}$ This implies that the genetic profile of this gene in the Negritos was significantly differentiated from the rest of the populations. CDH13 encodes for protein T-Cadherin, which belongs to the cadherin superfamily of the transmembrane proteins that mediate calcium-dependent intercellular adhesion, is the receptor for the high-molecular-weight adiponectin expressed in the vasculature ${ }^{40}$ and cardiac myocytes. ${ }^{41}$ Genetic variation of this gene has been shown to influence metabolic outcome and possibly provides atherogenesis resistance. ${ }^{39} 4243$ Adiponectin plays a crucial role in the metabolic regulation of obesity, insulin sensitivity and atherosclerosis and several studies have indicated its antiatherogenic properties. ${ }^{44}$ A recent study reported that minor allele of rs12051272 revealed a considerable association with a more favourable metabolic profile, including higher insulin sensitivity, HDL-c, lower DBP, FPG and TG concentrations. ${ }^{42}$ Given the phenotypic observations and the role of this gene, we postulate a plausibility of CDH13 regulating the phenotype and could explain the findings observed in our study whereby despite fulfilling criteria for MS, these Negritos subjects did not exhibit augmented inflammatory or endothelial activation status when compared with their non-MS counterparts. Future studies exploring expressions of genetic variants of $\mathrm{CDH} 13$ in these Negritos cohorts could further shed light on influences of this gene on atherogenesis.

Regression analysis has shown that the Negritos independently predict for $\mathrm{Lp}(\mathrm{a})$ in this study. This finding implies that despite hunter-gatherers get more exercise, putting them at a lower risk for heart disease and relatively isolated from modernisation, suggests that serum $\mathrm{Lp}(\mathrm{a})$ concentrations are not influenced by diet or lifestyle, but more significantly by variants in the $L P A$ gene. This is in parallel with previous studies that exhibited a significant difference in $\mathrm{Lp}(\mathrm{a})$ levels among various populations including Asian Indian, Chinese, non-Hispanic and blacks. ${ }^{45}{ }^{46}$ Studies have demonstrated the presence of LPA gene polymorphisms primarily determining levels of $\operatorname{Lp}(\mathrm{a})$, without significant dietary or environmental effects. ${ }^{47}$ Further studies are warranted to identify common polymorphisms in LPA gene among Negritos and other OA tribes, which would further validate these inferences.

The main constraint of this study was the small sample size of the Negrito group, which prevented us from comparing between ethnicities. Although the sample size included was enough to reject the null hypothesis, a larger sample size could provide a better representation of the Negrito population. However, it should be reiterated that Negrito represents only $3 \%$ of the total OA population in Malaysia and an even smaller percentage of that embodies the inland dwellers who are not subjected to urbanisation. This along with major physical problems accessing all these remote tribes prevented us from having a larger sample to analyse.

\section{CONCLUSION}

This study highlights several key findings that provide further insights into the metabolic differences between the inland-living aboriginal group, Negritos, in Malaysia and urbanised Malays in Malaysia. First, MS and non-MS Negritos failed to show differences in biomarkers of coronary risks as established by previous reports. Although the small Negrito sample could be a contributor to this observation, genetic or lifestyle influence cannot be ruled out. This warrants further studies to confirm these observations and, if replicated, paves way to future research to understand the mechanism behind this discrepancy. Second, Negritos independently predicting serum Lp(a) concentrations suggests a genetic influence that surpasses any form of impact by diet and lifestyle, which needs further exploration. Therefore, future studies to identify common variants of $L P A$ gene among this group and to extend such research to other Aborigine tribes would further improve our understanding of interaction between gene and phenotypic expression of $\mathrm{Lp}(\mathrm{a})$.

Acknowledgements The authors would like to express their appreciation to the Centre for Pathology Diagnostic and Research Laboratories (CPDRL) of Faculty of Medicine, Universiti Teknologi MARA for providing the facilities to conduct this research and all the subjects who voluntarily participated in this study. The authors gratefully acknowledge Roche Diagnostics, Malaysia for their support. 
Contributors NAMM collected the subjects, performed the experiments, analysed the samples and data, prepared the tables, wrote the manuscript and reviewed drafts of the paper. SSM collected the subjects, analysed the samples and reviewed drafts of the paper. AMI collected the subjects, validated results and reviewed drafts of the paper. FMN and SAS collected the subjects and reviewed drafts of the paper. HMN collected the subjects, contributed reagents and materials and reviewed drafts of the paper. KY contributed reagents and materials, drafted the manuscript and reviewed the draft of the paper. TAR conceived and designed the experiments, collected the subjects, contributed reagents and materials, wrote the manuscript and reviewed drafts of the paper. BPH conceived and designed the experiments, collected the subjects, validated results, contributed reagents and materials and reviewed drafts of the paper.

Funding The Ministry of Higher Education Malaysia under Fundamental Research Grant Scheme (Grant code: 600-RMI/FRGS 5/3 (136/2014)). The Ministry of Higher Education Malaysia under Long Term Research Grant Scheme (Grant code: 600-RMI/LRGS 5/3 (2/2011)-2).

Competing interests None declared.

Patient consent Obtained.

Ethics approval Universiti Teknologi MARA and Department of Orang Asli Development.

Provenance and peer review Not commissioned; externally peer reviewed.

Data sharing statement № additional data available.

Open access This is an open access article distributed in accordance with the Creative Commons Attribution Non Commercial (CC BY-NC 4.0) license, which permits others to distribute, remix, adapt, build upon this work non-commercially, and license their derivative works on different terms, provided the original work is properly cited, appropriate credit is given, any changes made indicated, and the use is non-commercial. See: http://creativecommons.org/licenses/by-nc/4.0/.

\section{REFERENCES}

1. Phipps ME, Chan KK, Naidu R, et al. Cardio-metabolic health risks in indigenous populations of Southeast Asia and the influence of urbanization. BMC Public Health 2015;15:1-8.

2. Masron T, Masami F, Ismail N. Orang Asli in Peninsular Malaysia: population, spatial distribution and socio-economic condition. J Ritsumeikan Soc Sci Humanit 2013;6:75-115.

3. Lim YA, Romano N, Colin N, et al. Intestinal parasitic infections amongst Orang Asli (indigenous) in Malaysia: has socioeconomic development alleviated the problem? Trop Biomed 2009;26:110-22.

4. Weng X, Liu Y, Ma J, et al. An urban-rural comparison of the prevalence of the metabolic syndrome in Eastern China. Public Health Nutr 2007;10:131-6.

5. Prabhakaran D, Chaturvedi V, Shah $P$, et al. Differences in the prevalence of metabolic syndrome in urban and rural India: a problem of urbanization. Chronic IIIn 2007;3:8-19.

6. Ordovas JM. Genetic links between diabetes mellitus and coronary atherosclerosis. Curr Atheroscler Rep 2007;9:204-10.

7. Zambon A, Pauletto P, Crepaldi G. Review article: the metabolic syndrome-a chronic cardiovascular inflammatory condition. Aliment Pharmacol Ther 2005;22:20-3.

8. Eckel RH, Grundy SM, Zimmet PZ. The metabolic syndrome. Lancet 2005;365:1415-28.

9. Mohamud WN, Ismail A, Khir AS, et al. Prevalence of metabolic syndrome and its risk factors in adult Malaysians: results of a nationwide survey. Diabetes Res Clin Pract 2012;96:91-7.

10. Fuentes E, Fuentes F, Vilahur G, et al. Mechanisms of chronic state of inflammation as mediators that link obese adipose tissue and metabolic syndrome. Mediators Inflamm 2013;2013:1-11.

11. Weisberg SP, McCann D, Desai M, et al. Obesity is associated with macrophage accumulation in adipose tissue. J Clin Invest 2003;112:1796-808.

12. Mottillo S, Filion KB, Genest J, et al. The metabolic syndrome and cardiovascular risk a systematic review and meta-analysis. J Am Coll Cardiol 2010;56:1113-32.

13. Bermúdez V, Rojas J, Salazar J, et al; Variations of lipoprotein (a. 2013. Levels in the Metabolic Syndrome: A Report from the Maracaibo City Metabolic Syndrome Prevalence Study, 2013.

14. Loscalzo J. Lipoprotein(a). A unique risk factor for atherothrombotic disease. Arteriosclerosis 1990;10:672-9.

15. Loscalzo J, Weinfeld M, Fless GM, et al. Lipoprotein(a), fibrin binding, and plasminogen activation. Arteriosclerosis: An Official Journal of the American Heart Association, Inc. 1990;10:240-5.
16. Lim KG, Cheah WK. A review of metabolic syndrome research in Malaysia. Med J Malaysia 2016;71:20-8.

17. Alberti SG, Zimmet P, Shaw J, et al. The IDF consensus worldwide definition of the metabolic syndrom. 2006 https://www.idf.org/ webdata/docs/IDF_Meta_def_final.pdf.

18. Dupont WD, Plummer WD. Power and sample size calculations. Control Clin Trials 1990;11:116-28.

19. Ashari LS, Mitra AK, Rahman TA, et al. Prevalence and risk factors of metabolic syndrome among an endangered tribal population in Malaysia using harmonized IDF criteria. Int J Diabetes Dev Ctries 2016;36:352-8.

20. Mohamud WN, Ismail AA, Sharifuddin A, et al. Prevalence of metabolic syndrome and its risk factors in adult Malaysians: results of a nationwide survey. Diabetes Res Clin Pract 2011;91:239-45.

21. Friedewald WT, Levy RI, Fredrickson DS. Estimation of the concentration of low-density lipoprotein cholesterol in plasma, without use of the preparative ultracentrifuge. Clin Chem 1972;18:499-502.

22. Alberti KG, Eckel RH, Grundy SM, et al. Harmonizing the metabolic syndrome: a joint interim statement of the international diabetes federation task force on epidemiology and prevention; national heart, lung, and blood institute; american heart association; world heart federation; international atherosclerosis society; and international association for the study of obesity. Circulation 2009;120:1640-5.

23. Isomaa B, Almgren $\mathrm{P}$, Tuomi $\mathrm{T}$, et al. Cardiovascular morbidity and mortality associated with the metabolic syndrome. Diabetes Care 2001;24:683-9.

24. Lakka H-M. The metabolic syndrome and total and cardiovascular disease mortality in middle-aged men. JAMA 2002;288:2709-16.

25. Odegaard AO, Jacobs DR, Sanchez OA, et al. Oxidative stress, inflammation, endothelial dysfunction and incidence of type 2 diabetes. Cardiovasc Diabetol 2016;15:51.

26. Tuttolomondo A, Pecoraro R, Casuccio A, et al. Peripheral frequency of CD4+ CD28- cells in acute ischemic stroke: relationship with stroke subtype and severity markers. Medicine 2015;94:1-8.

27. Subirana I, Fitó M, Diaz O, et al. Prediction of coronary disease incidence by biomarkers of inflammation, oxidation, and metabolism. Sci Rep 2018;8:3191.

28. Signorelli SS, Anzaldi M, Libra M, et al. Plasma levels of inflammatory biomarkers in peripheral arterial disease. Angiology 2016;67:870-4

29. Cheng $Y X$, Chong CP, Kiew CF, et al. An assessment of health and social-economic status among Lanoh ethnic sub-group of Orang Asli (indigenous peoples) in Air Bah I village, state of Perak, Malaysia. J Appl Pharm Sci 2014;4:32-7.

30. Tuan Abdul Aziz TA, Teh LK, Md Idris MH, et al. Increased risks of cardiovascular diseases and insulin resistance among the Orang Asli in Peninsular Malaysia. BMC Public Health 2016;16:284.

31. Weiss TW, Arnesen H, Seljeflot I. Components of the interleukin-6 transsignalling system are associated with the metabolic syndrome, endothelial dysfunction and arterial stiffness. Metabolism 2013;62:1008-13.

32. Brake DK, Smith EO, Mersmann H, et al. ICAM-1 expression in adipose tissue: effects of diet-induced obesity in mice. Am J Physiol Cell Physiol 2006;291:C1232-9.

33. Tang L, Peng H, Xu T, et al. Association of biomarkers of inflammation with dyslipidemia and its components among Mongolians in China. PLoS One 2014;9:e89023.

34. Mazidi M, Toth PP, Banach M. C-reactive protein is associated with prevalence of the metabolic syndrome, hypertension, and diabetes mellitus in US adults. Angiology 2018;69:438-42.

35. Ferreira I, Hovind P, Schalkwijk CG, et al. Biomarkers of inflammation and endothelial dysfunction as predictors of pulse pressure and incident hypertension in type 1 diabetes: a 20 year life-course study in an inception cohort. Diabetologia 2018;61:231-41.

36. Fonseca FA, Izar MC. High-Sensitivity C-Reactive Protein and Cardiovascular disease across countries and ethnicities. Clinics 2016;71:235-42.

37. Tuttolomondo A, Di Raimondo D, Pecoraro R, et al. Atherosclerosis as an inflammatory disease. Curr Pharm Des 2012;18:4266-88.

38. Deng L, Hoh BP, Lu D, et al. The population genomic landscape of human genetic structure, admixture history and local adaptation in Peninsular Malaysia. Hum Genet 2014;133:1169-85

39. Liu X, Yunus Y, Lu D, et al. Differential positive selection of malaria resistance genes in three indigenous populations of Peninsular Malaysia. Hum Genet 2015;134:375-92.

40. Hebbard LW, Garlatti M, Young LJ, et al. T-cadherin supports angiogenesis and adiponectin association with the vasculature in a mouse mammary tumor model. Cancer Res 2008;68:1407-16.

41. Denzel MS, Scimia MC, Zumstein PM, et al. T-cadherin is critical for adiponectin-mediated cardioprotection in mice. J Clin Invest 2010;120:4342-52. 
42. Teng MS, Hsu LA, Wu S, et al. Association of CDH13 genotypes/ haplotypes with circulating adiponectin levels, metabolic syndrome, and related metabolic phenotypes: the role of the suppression effect. PLoS One 2015;10:e0122664-13.

43. Gao H, Kim YM, Chen P, et al. Genetic variation in $\mathrm{CDH} 13$ is associated with lower plasma adiponectin levels but greater adiponectin sensitivity in East Asian populations. Diabetes 2013;62:4277-83.

44. Matsuzawa Y, Funahashi T, Kihara S, et al. Adiponectin and metabolic syndrome. Arterioscler Thromb Vasc Biol 2004;24:29-33.
45. Banerjee D, Wong EC, Shin J, et al. Racial and ethnic variation in lipoprotein (a) levels among Asian Indian and Chinese Patients. $J$ Lipids 2011;2011:1-6.

46. Guyton JR, Dahlen GH, Patsch W, et al. Relationship of plasma lipoprotein $L p(a)$ levels to race and to apolipoprotein $B$. Arteriosclerosis 1985;5:265-72.

47. Tsimikas S, Hall JL. Lipoprotein(a) as a potential causal genetic risk factor of cardiovascular disease: a rationale for increased efforts to understand its pathophysiology and develop targeted therapies. J Am Coll Cardiol 2012;60:716-21. 